\title{
Coherence in Spontaneous Radiation Processes
}

\author{
R. H. DickE \\ Palmer Physical Laboratory, Princeton University, Princeton, New Jersey
}

(Received August 25, 1953)

\begin{abstract}
By considering a radiating gas as a single quantum-mechanical system, energy levels corresponding to certain correlations between individual molecules are described. Spontaneous emission of radiation in a transition between two such levels leads to the emission of coherent radiation. The discussion is limited first to a gas of dimension small compared with a wavelength. Spontaneous radiation rates and natural line breadths are calculated. For a gas of large extent the effect of photon recoil momentum on coherence is calculated. The effect of a radiation pulse in exciting "super-radiant" states is discussed. The angular correlation between successive photons spontaneously emitted by a gas initially in thermal equilibrium is calculated.
\end{abstract}

$I_{a}^{\mathrm{N}}$ the usual treatment of spontaneous radiation by a gas, the radiation process is calculated as though the separate molecules radiate independently of each other. To justify this assumption it might be argued that, as a result of the large distance between molecules and subsequent weak interactions, the probability of a given molecule emitting a photon should be independent of the states of other molecules. It is clear that this model is incapable of describing a coherent spontaneous radiation process since the radiation rate is proportional to the molecular concentration rather than to the square of the concentration. This simplified picture overlooks the fact that all the molecules are interacting with a common radiation field and hence cannot be treated as independent. The model is wrong in principle and many of the results obtained from it are incorrect.

A simple example will be used to illustrate the inadequacy of this description. Assume that a neutron is placed in a uniform magnetic field in the higher energy of the two spin states. In due course the neutron will spontaneously radiate a photon via a magnetic dipole transition and drop to the lower energy state. The probability of finding the neutron in its upper energy state falls exponentially to zero., ${ }^{1,2}$

If, now, a neutron in its ground state is placed near the first excited neutron (a distance small compared with a radiation wavelength but large compared with a particle wavelength and such that the dipole-dipole interaction is negligible), the radiation process would, according to the above hypothesis of independence, be unaffected. Actually, the radiation process would be strongly affected. The initial transition probability would be the same as before but the probability of finding an excited neutron would fall exponentially to one-half rather than to zero.

The justification for these assertions is the following: The initial state of the neutron system finds neutron 1 excited and neutron 2 unexcited. (It is assumed that the particles have nonoverlapping space functions, so that particle symmetry plays no role.) This initial state may be considered to be a superposition of the

\footnotetext{
${ }^{1}$ W. Heitler, The Quantum Theory of Radiation (Clarendon Press, Oxford, 1936), first edition, p. 112.

2 E. P. Wigner and V. Weisskopf, Z. Physik 63, 54 (1930).
}

triplet and singlet states of the particles. The triplet state is capable of radiating to the ground state (triplet) but the singlet state will not couple with the triplet system. Consequently, only the triplet part is modified by the coupling with the field. After a long time there is still a probability of one-half that a photon has not been emitted. If, after a long period of time, no photon has been emitted, the neutrons are in a singlet state and it is impossible to predict which neutron is the excited one.

On the other hand, if the initial state of the two neutrons were triplet with $s=1, m_{s}=0$ namely a state with one excited neutron, a photon would be certain to be emitted and the transition probability would be just double that for a lone excited neutron. Thus, the presence of the unexcited neutron in this case doubles the radiation rate.

In recent years the excitation of correlated states of atomic radiating systems with the subsequent emission of spontaneous coherent radiation has become an important technique for nuclear magnetic resonance research. ${ }^{3}$ The description usually given of this process is a classical one based on a spin system in a magnetic field. The purpose of this note is to generalize these results to any system of radiators with a magnetic or electric dipole transition and to see what effects, if any, result from a quantum mechanical treatment of the radiation process. Most of the previous work ${ }^{4}$ was quite early and not concerned with the problems being considered here. In a subsequent article to be published in the Review of Scientific Instruments some of these results will be applied to the problem of instrumentation for microwave spectroscopy.

In this treatment the gas as a whole will be considered as a single quantum-mechanical system. The problem will be one of finding those energy states representing correlated motions in the system. The spontaneous emission of coherent radiation will accompany transitions between such levels. In the first problem to be considered the gas volumes will be assumed to have

3 E. L. Hahn, Phys. Rev. 77, 297 (1950); 80, 580 (1950).

${ }^{4}$ E.g., W. Pauli, Handbuch der Physik (Springer, Berlin, 1933), Vol. 24, Part I, p. 210; G. Wentzel, Handbuch der Physik (Springer, Berlin, 1933), Vol. 24, Part I, p. 758. 
dimensions small compared with a radiation wavelength. This case, which is of particular importance for nuclear magnetic resonance experiments and some microwave spectroscopic applications, is treated first quantum mechanically and then semiclassically, the radiation process being treated classically. A classical model is also described. In the next case to be considered the gas is assumed to be of large extent. The effect of molecular motion on coherence and the effect on coherence of the recoil momentum accompanying the emission of a photon are discussed. Finally, the two principal methods of exciting coherent states by the absorption of photons from an intense radiation pulse or the emission of photons by the gas are discussed. Calculations of these two effects are made for the gas system initially in thermal equilibrium. The effect of photon emission on inducing coherence is discussed as a problem in the angular correlation of the emitted photons.

\section{DIPOLE APPROXIMATION}

The first problem to be considered is that of a gas confined to a container the dimensions of which are small compared with a wavelength. It is assumed that the walls of the container are transparent to the radiation field. In order to avoid difficulties arising from collision broadening it will be assumed that collisions do not affect the internal states of the molecules. It will be assumed that the transition under question takes place between two nondegenerate states of the molecule. The assumption of nondegeneracy is made in order to limit the scope of the problem to its bare essentials. It might be assumed that nondegenerate states are present as a result of a uniform static electric or magnetic field acting on the gas. Actually, for many of the questions being discussed it is not essential that the degeneracies be split. Also, it will be assumed that there is insufficient overlap in the wave functions of separate molecules to require that the wave functions be symmetrized.

Since it is assumed that internal coordinates of the individual molecules are unaffected by collisions and but two internal states are involved for each molecule, the wave function for the gas may be written conveniently in a representation diagonal in the center-ofmass coordinates and the internal energies of the molecules. The internal energy coordinate takes on only two values. Omitting for the moment the radiation field, the Hamiltonian for an $n$ molecule gas can be written

$$
H=H_{0}+E \sum_{j=1}^{n} R_{j 3}
$$

where $E=\hbar \omega=$ molecular excitation energy. Here $H_{0}$ acts on the center-of-mass coordinates and represents the translational and intermolecular interaction energies of the gas. $E R_{j 3}$ is the internal energy of the $j$ th molecule and has eigenvalues $\pm \frac{1}{2} E . H_{0}$ and all the $R_{j 3}$ commute with each other. Consequently, energy eigenfunctions may be ${ }^{\text {s }}$ chosen to be simultaneous eigenfunctions of $H_{0}, R_{13}, R_{23}, \cdots, R_{n 3}$.
Let a typical energy state be written as

$$
\psi_{g m}=U_{g}\left(\mathbf{r}_{1} \cdots \mathbf{r}_{n}\right)[++-+\cdots] \text {. }
$$

Here $\mathbf{r}_{1} \cdots \mathbf{r}_{n}$ designates the center-of-mass coordinates of the $n$ molecules, and + and - symbols represent the internal energies of the various molecules. If the number of + and - symbols are denoted by $n_{+}$and $n_{-}$, respectively, then $m$ is defined as

$$
\begin{aligned}
m & =\frac{1}{2}\left(n_{+}-n_{-}\right), \\
n & =n_{+}+n_{-}=\text {number of gaseous molecules. }
\end{aligned}
$$

If the energy of motion and mutual interaction of the molecules is denoted by $E_{g}$, then the total energy of the system is

$$
E_{g m}=E_{g}+m E \text {. }
$$

It is evident that the index $m$ is integral or half-integral depending upon whether $n$ is even or odd. Because of the various orders in which the + and - symbols can be arranged, the energy $E_{g m}$ has a degeneracy

$$
\frac{n !}{\left(\frac{1}{2} n+m\right) !\left(\frac{1}{2} n-m\right) !} \text {. }
$$

This degeneracy has its origin in the internal coordinates only.

In addition, the wave function may have additional degeneracy from the center-of-mass coordinates. It should be noted in this connection that the degeneracy of the total wave function will depend upon whether or not the molecules are regarded as distinguishable or not.

If the molecules are indistinguishable, the symmetry of $U_{g}$ will depend upon the symmetries of the wave function under interchanges of internal coordinates. For example, the states with all molecules excited are symmetric under an interchange of the internal coordinates of any two molecules. Consequently, for these states $U_{g}$ must be symmetric for Bose molecules and antisymmetric for Fermi molecules. The limitations of symmetry are normally without physical significance as it is assumed that the gas is of such low density that the various molecules have nonoverlapping wave functions.

Of the Hamiltonian equation (1), $H_{0}$ operates on the center-of-mass coordinates only and gives

$$
H_{0} U_{g}=E_{g} U_{g}
$$

whereas $R_{j 3}$ operates on the plus or minus symbol in the $j$ th place corresponding to the internal energy of the $j$ th molecule. Except for the factor $\frac{1}{2}$, it is analogous to one of the Pauli spin operators. As operators similar to the other two Pauli operators are also needed in this development, the properties of all three are listed here.

$$
\begin{gathered}
j \\
\downarrow \\
R_{j 1}[\cdots \pm \cdots]=\frac{1}{2}[\cdots \mp \cdots], \\
R_{j 2}[\cdots \pm \cdots]= \pm \frac{1}{2} i[\cdots \mp \cdots] \\
R_{j 3}[\cdots \pm \cdots]= \pm \frac{1}{2}[\cdots \pm \cdots] .
\end{gathered}
$$


It is also convenient to define the operators

$$
R_{k}=\sum_{j=1}^{n} R_{j k}, \quad k=1,2,3,
$$

and the operator

$$
R^{2}=R_{1}^{2}+R_{2}^{2}+R_{3}^{2} \text {. }
$$

In this notation the Hamiltonian becomes

and

$$
H=H_{0}+E R_{3},
$$

$$
R_{3} \psi_{g m}=m \psi_{g m}
$$

To complete the description of the dynamical system, there must be added to the Hamiltonian that of the radiation field and the interaction term between field and the molecular system.

For the purpose of definiteness the ineraction of a molecule with the electromagnetic field will be assumed to be electric dipole. The main results are actually independent of the type of coupling. The interaction energy of the $j$ th molecule with the electromagnetic field can be written as

$$
-\mathbf{A}\left(\mathbf{r}_{j}\right) \cdot \sum_{k=1}^{N-1} \frac{e_{k}}{m_{k} c} \mathbf{P}_{k}
$$

Here the configuration coordinates of the molecule are taken to be the center-of-mass coordinates and the coordinates relative to the center of mass of any $N-1$ of the $N$ particles which constitute the $j$ th molecule. $e_{k}$ and $m_{k}$ are the charge and mass of the $k$ th particle, and $\mathbf{P}_{\mathrm{k}}$ is the momentum conjugate to the position of the $k$ th particle relative to the center of mass. The molecule is assumed electrically neutral.

Since $\mathbf{P}_{k}$ is an odd operator, it has only off-diagonal elements in a representation with internal energy diagonal. Hence the general form of Eq. (12) is

$$
-\mathbf{A}\left(\mathbf{r}_{j}\right) \cdot\left(\mathbf{e}_{1} R_{j 1}+\mathbf{e}_{2} R_{j 2}\right) .
$$

$\mathbf{e}_{1}$ and $\mathbf{e}_{2}$ are constant real vectors the same for all molecules. The total interaction energy then becomes

$$
H_{1}=-\sum_{j} \mathrm{~A}\left(\mathbf{r}_{j}\right) \cdot\left(\mathbf{e}_{1} R_{j 1}+\mathbf{e}_{2} R_{j 2}\right) .
$$

Since the dimensions of the gas cell are small compared with a wavelength, the dependence of the vector potential on the center of mass of the molecules can be omitted and the interaction energy (12) becomes

$$
H_{1}=-\mathbf{A}(0) \cdot\left(\mathbf{e}_{1} R_{1}+\mathbf{e}_{2} R_{2}\right) .
$$

Since the interaction term Eq. (15) does not contain the center-of-mass coordinates, the selection rule on the molecular motion quantum number $g$ is $\Delta g=0$. Consequently there is no Doppler broadening of the transition frequency. This results solely from the small size of the gas container. ${ }^{5}$

The operators $R_{1}, R_{2}$, and $R_{3}$, apart from a factor of $\hbar$, obey the same commutation relations as the three

\footnotetext{
${ }^{5}$ R. H. Dicke, Phys. Rev. 89, 472 (1953).
}

components of angular momentum. Consequently, the interaction operator Eq. (15) obeys the selection rule $\Delta m= \pm 1$. In general, it has nonvanishing matrix elements between a given state Eq. (2) and a large number of states with $\Delta m= \pm 1$. In order to simplify the calculation of spontaneous radiation transitions, it is desirable that a set of stationary states be selected in such a way that the interaction term has matrix elements joining a given state with, at most, one state of higher and lower energy, respectively. Because of the very close analogy between this formalism and that of a system of particles of spin $\frac{1}{2}$, known results can be taken over from the spin formalism.

In a manner similar to an angular momentum formalism, ${ }^{6}$ the operations $H$ and $R^{2}$ commute; consequently, stationary states can be chosen to be eigenstates of $R^{2}$. These new states are linear combinations of the states of Eq. (2). The operator $R^{2}$ has eigenvalues $r(r+1) . r$ is integral or half-integral and positive, such that

$$
|m| \leqslant r \leqslant \frac{1}{2} n .
$$

The eigenvalue $r$ will be called the "cooperation number" of the gas. Denote the new eigenstates by

Here

$$
\psi_{g m r} \text {. }
$$

$$
\begin{aligned}
& H \psi_{g m r}=\left(E_{g}+m E\right) \psi_{g m r}, \\
& R^{2} \psi_{g m r}=r(r+1) \psi_{g m r} .
\end{aligned}
$$

The degeneracy of the stationary states is not completely removed by introducing $R^{2}$. The state $(g, m, r)$ has a degeneracy

$$
\frac{n !(2 r+1)}{\left(\frac{1}{2} n+r+1\right) !\left(\frac{1}{2} n-r\right) !} \text {. }
$$

The complete set of eigenstates $\psi_{g m r}$ may be specified in the following way: the largest value of $m$ and $r$ is

$$
r=m=\frac{1}{2} n \text {. }
$$

This state is nondegenerate in the internal coordinates and may be written as

$$
\psi_{g, \frac{1}{2} n, \frac{1}{2} n}=U_{g} \cdot[++\cdots+] .
$$

All the states with this same value of $r=\frac{1}{2} n$, but with different values of $m$, are nondegenerate also and may be generated $\mathrm{as}^{7}$

$$
\psi_{g m r}=\left[\left(R^{2}-R_{3}^{2}-R_{3}\right)^{-\frac{1}{2}}\left(R_{1}-i R_{2}\right)\right]^{r-m} \psi_{g r r} .
$$

The operator $R_{1}-i R_{2}$ reduces the $m$ index by unity every time it is applied and the fractional power operator is to preserve the normalization of the wave function. ${ }^{8}$ The fractional power operator is defined as having positive eigenvalues only.

${ }^{6}$ E. U. Condon and G. H. Shortley, The Theory of Atomic Spectra (Cambridge University Press, Cambridge, 1935), pp. 45-49.

${ }^{7}$ See reference 6, p. 48, Eq. (3).

${ }^{8}$ See reference 6, p. 48. 
The state $\psi_{g, \frac{1}{2} n-1, \frac{1}{2} n}$ is one of $n$ states with this value of $m$. The remaining $n-1$ states should be chosen to be orthogonal to this state, orthogonal to each other, and normalized. Since these remaining $n-1$ states are not states of $r=\frac{1}{2} n$, they must be states of $r=\frac{1}{2} n-1$, the only other possibility. Again the complete set of states with this value of $r$ can be generated using Eq. (21), where now $r=\frac{1}{2} n-1$, and the operator in Eq. (21) is applied to each of the $n-1$ orthogonal states of $r=m=\frac{1}{2} n-1$. This procedure can be repeated until all possible values of $r$ are exhausted, in which case all the stationary states have been defined.

With this definition of the stationary states, the interaction energy operator has matrix elements joining a given state of the gas to but two other states. Aside from the factor involving the radiation field operator, the matrix elements of the interaction energy may be written $^{8}$

$$
\begin{aligned}
\left(g, r, m\left|\mathbf{e}_{1} R_{1}+\mathbf{e}_{2} R_{2}\right| g, r, m \mp 1\right) \\
=\frac{1}{2}\left(\mathbf{e}_{1} \pm i \mathbf{e}_{2}\right)[(r \pm m)(r \mp m+1)]^{\frac{1}{2}} .
\end{aligned}
$$

Transition probabilities will be proportional to the square of the matrix elements. In particular, the spontaneous radiation probabilities will be

$$
I=I_{0}(r+m)(r-m+1) .
$$

Here, by setting $r=m=\frac{1}{2}$, it is evident that $I_{0}$ is the radiation rate of a gas composed of one molecule in its excited state. $I_{0}$ has the value ${ }^{9}$

$$
\begin{aligned}
I_{0}=\frac{4 \omega^{2}}{3} \frac{-}{c}\left|\left(\sum_{k} \frac{e_{k} \mathbf{P}_{k}}{m_{k} c}\right)_{+-}\right|^{2}=\frac{1 \omega^{2}}{3}-\left|\mathbf{e}_{1}-i \mathbf{e}_{2}\right|^{2} \\
=\frac{1 \omega^{2}}{3 c}\left(e_{1}^{2}+e_{2}^{2}\right) .
\end{aligned}
$$

If $m=r=\frac{1}{2} n$ (i.e., all $n$ molecules excited),

$$
I=n I_{0} .
$$

Coherent radiation is emitted when $r$ is large but $|m|$ small. For example, for even $n$ let

$$
r=\frac{1}{2} n, \quad m=0 ; \quad I=\frac{1}{2} n\left(\frac{1}{2} n+1\right) I_{0} .
$$

This is the largest rate at which a gas with an even number of molecules can radiate spontaneously. It should be noted that for large $n$ it is proportional to the square of the number of molecules.

Because of the fact that with the choice of stationary states given by Eq. (21) a given state couples with but one state of lower energy, this radiation rate [Eq. (27)], is an absolute maximum. Any superposition state will radiate at the rate

$$
\begin{aligned}
I=I_{0} \sum_{r, m} P_{r, m}(r+m)(r-m+1) & \\
& =I_{0}\left\langle\left(R_{1}+i R_{2}\right)\left(R_{1}-i R_{2}\right)\right\rangle,
\end{aligned}
$$

where $P_{r, m}$ is the probability of being in the state $r, m$.

\footnotetext{
${ }^{9}$ Reference 1, p. 106.
}

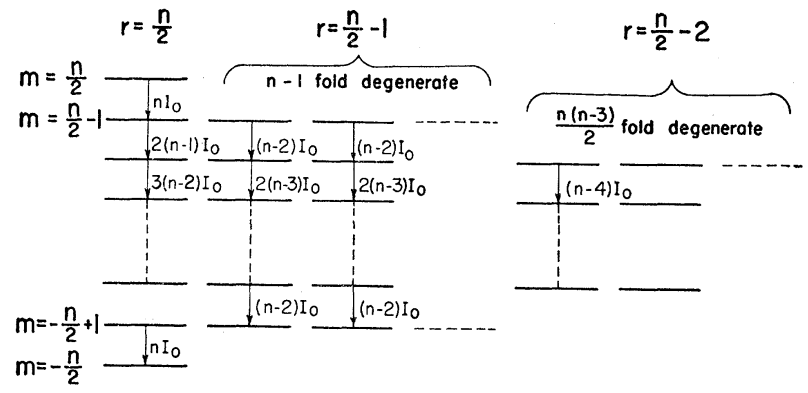

Frg. 1. Energy level diagram of an $n$-molecule gas, each molecule having 2 nondegenerate energy levels. Spontaneous radiation rates are indicated. $E_{m}=m E$.

There are no interference terms. Consequently, no superposition state can radiate more strongly than Eq. (27). An energy level diagram which shows the relative magnitudes of the various radiation probabilities is given in Fig. 1.

States with a low "cooperation number" are also highly correlated but in such a way as to have abnormally low radiation rates. For example, a gas in the state $r=m=0$ does not radiate at all. This state, which exists only for an even number of molecules, is analogous to a classical system of an even number of oscillators swinging in pairs oppositely phased.

The energy trapping which results from the internal scattering of photons by the gas appears naturally in the formalism. As an example, consider an initial state of the gas for which one definite molecule, and only this molecule, is excited. The gas at first radiates at the normal incoherent rate for a short time and thereafter fails to radiate. The probability of a photon's being emitted during the radiating period is $1 / n$. These results follow from the fact that the assumed state is a linear superposition of the various states with $m=1-n / 2$, and that $1 / n$ is the probability of being in the state $r=\frac{1}{2} n$. The probability that the energy will be "trapped" is $(n-1) / n$. This is analogous to the radiation by a classical oscillator when $n-1$ similar unexcited oscillators are near. The solution of this classical problem shows that only $1 / n$ of the excitation energy is radiated. The remainder appears in nonradiating normal modes of the system.

For want of a better term, a gas which is radiating strongly because of coherence will be called "superradiant." There are two obvious ways in which a "super-radiant" state may be excited. First, if all the molecules be excited, the gas is in the state characterized by

$$
r=m=\frac{1}{2} n \text {. }
$$

As the system radiates it passes to states of lower $m$ with $r$ unchanged. This will take the system to the "super-radiant" region $m \sim 0$.

Another way in which such a state can be excited is to start with the gas in its ground state,

$$
r=-m=\frac{1}{2} n \text {, }
$$


and irradiate it with a pulse of radiation. ${ }^{9 \mathrm{a}}$ If the pulse is sufficiently intense, the system is lifted to energy states with $m \sim 0$ but with $r$ unchanged, and these states are "super-radiant."

Although the "super-radiant" states have abnormally large spontaneous radiation rates, the stimulated emission rate is normal. For example, with the system in the state $m, r$, the stimulated emission rate is proportional to

$$
(r+m)(r-m+1)-(r+m+1)(r-m)=2 m .
$$

With $m>0$ this is the normal incoherent stimulated emission rate. For $m<0$ this becomes the negative of the incoherent absorption rate.

As has been pointed out, the pulse technique for exciting "super-radiant" states is commonly used in nuclear magnetic resonance experiments. Here there is one important point that needs clarification, however. Instead of starting in the highly organized state given by Eq. (30) the pulse is applied to a system that is in thermal equilibrium at high temperatures. For example, if the system be a set of proton spins, the energy necessary to turn a spin over in the magnetic field may be about

$$
E \sim 10^{-5} k T .
$$

Under these conditions the two spin states of the proton are very nearly equally populated and it might be expected that thermal equilibrium would imply a badly disorganized system. The randomness in the initial state does not imply, however, complete randomness in $m$ and $r$. For a gas with $n$, large states of low $r$ have a high degeneracy. These states have a high statistical weight and are favored. However, Eq. (16) sets a lower bound on $r$ for any $m$. The result is a relatively small range of values of $m$ and $r$. For a system with $n$ molecules in thermal equilibrium the mean square deviation from the mean of $m$ is

$$
n / 4-\bar{m}^{2} / n \text {. }
$$

Here $\bar{m}$ is the mean of $m$ and is for high temperatures equal to

$$
\bar{m}=-\frac{1}{4} n E / k T .
$$

For a definite value of $m$ the mean value of $r(r+1)$ is

$$
m^{2}+\frac{1}{2} n
$$

and the mean square deviation is

$$
\frac{1}{4} n^{2}-m^{2} \text {. }
$$

The expression (32)-(35) may be easily derived using the density matrix formalism assuming the appropriate statistical ensemble.

It is hence clear that if

$$
\bar{m}^{2} \gg n \gg 1,
$$

the percentage deviation from the mean of $m$ is small,

9a See F. Bloch and I. I. Rabi, Revs. Modern Phys. 17, 237 (1945), for a discussion of the effect of a pulse on the analogous spin- $\frac{1}{2}$ system. that the percent deviation from the mean of $r(r+1)$ is small, and that the mean of $r(r+1)$ is approximately the smallest value compatible with the mean value of $m$. Thus, in the case of a gas system at high temperature, for sufficiently large $n$, values of $m$ and $r$ cluster to such an extent that the system may be considered as approximately in a state of definite $r=m=-n E / 4 k T$. If this gas is excited by a pulse of the proper intensity to excite states $m \sim 0$, the radiation rate after the pulse is approximately

$$
I \cong I_{0} r(r+1) \cong I_{0} n^{2}(E / 4 k T)^{2},
$$

which is proportional to $n^{2}$ and hence coherent. A better calculation good for all temperatures gives the result [see Eq. (78) with $\theta=90^{\circ}$ ]

$$
I=\frac{1}{4} I_{0} n(n-1) \tanh ^{2}(E / 2 k T)+\frac{1}{2} n I_{0} .
$$

\section{SEMICLASSICAL TREATMENT}

For the spontaneous radiation from super-radiant states $(m \sim 0)$ a semiclassical treatment is generally adequate. This method, which is a generalization of the well-known picture used in describing radiation from a nuclear spin system, ${ }^{10}$ treats the molecular systems quantum mechanically but calculates the radiation process classically. In the following calculation the gas system will be assumed to be excited by a radiation pulse, which excites it from thermal equilibrium to a set of super-radiant states. To calculate the radiation rate, the expectation value of the electric dipole moment is treated as a classical dipole. When the gas contains a large number of molecules the dipole moment of the gas as a whole should be given by the sum of the expectation values of the individual dipole moments.

In thermal equilibrium the gas may be considered as having $n_{-}$molecules in the ground state and $n_{+}$molecules in the excited state. A molecule which is initially in its ground state is assumed to be thrown into a superposition state of + and - by the radiation pulse. It is assumed that there is a unity probability ratio. The internal part of the wave function of the molecules after the pulse is given by

$\psi_{+}=\frac{1}{\sqrt{2}}\left\{[+] \exp \left(\begin{array}{c}\omega \\ -i-t \\ 2\end{array}\right)+[-] \exp i\left(\begin{array}{l}\omega \\ -t+\delta\end{array}\right)\right\}$.

This is the most general form for $\psi_{+}$apart from a possible multiplication phase factor. Here $\delta$ is a phase given by the phase of the exciting pulse. In a similar way a molecule in the excited state has its wave function converted to

$$
\psi_{-}=\frac{1}{\sqrt{2}}\left\{[-] \stackrel{\omega}{\exp i-t-[+]} \underset{2}{\exp }\left(\begin{array}{c}
-\underset{\omega}{-}-t-i \delta \\
2
\end{array}\right)\right\} .
$$

Instead of calculating the expectation value of the electric dipole moment it is more convenient to calculate the expectation value of the polarization current of the

\footnotetext{
${ }^{10}$ F. Bloch, Phys. Rev. 70, 460 (1946).
} 
$j$ th molecule given by

$$
\begin{aligned}
\left(\sum_{k=1}^{N-1} \frac{e_{k} \mathbf{P}_{k}}{m_{k}}\right) & =c\left\langle\mathbf{e}_{1} R_{j 1}+\mathbf{e}_{2} R_{j 2}\right\rangle \\
& = \pm \frac{1}{2} c\left[\mathbf{e}_{1} \cos (\omega t+\delta)+\mathbf{e}_{2} \sin (\omega t+\delta)\right] .
\end{aligned}
$$

The plus sign is obtained from the plus state, Eq. (38), and the negative sign from Eq. (39). Note the oscillating time dependence which results from the states being energy-superposition states. The polarization current for the gas as a whole is then

$$
\mathbf{j}=\left(n_{+}-n_{-}\right)(c / 2)\left[\mathbf{e}_{1} \cos (\omega t+\delta)+\mathbf{e}_{2} \sin (\omega t+\delta)\right] \text {. }
$$

The radiation rate calculated classically is then ${ }^{11}$

$$
I=\frac{2}{3} \frac{\omega^{2}}{c_{3}}\left|\mathbf{J}^{2}\right|=\frac{1}{12} \frac{\omega^{2}}{c}\left(n_{+}-n_{-}\right)^{2}\left(e_{1}^{2}+e_{2}^{2}\right) .
$$

In thermal equilibrium $n_{+} / n_{-}=\exp (-E / k T)$, from which

$$
n_{+}-n_{-}=n \tanh (E / 2 k T) .
$$

Substituting into Eq. (42) gives the classical radiation rate

$$
I=\frac{1}{12} \frac{\omega^{2}}{c} n^{2}\left(e_{1}^{2}+e_{2}^{2}\right) \tanh ^{2}\left(\frac{E}{2 k T}\right) .
$$

This may be compared with the quantum-mechanical result [Eq. (37a) and Eq. (25)]. For large $n$ the two results are equal.

\section{CLASSICAL MODEL}

When the gas is in a state of definite "cooperation number" $r$ which has a very large value, it is possible to represent it in its interaction with the electromagnetic field by a simple classical model. The energy-level spacing and the matrix elements joining adjacent levels are similar to those of a rotating top of large angular momentum and carrying an electric dipole moment. The details depend upon $\mathbf{e}_{1}$ and $\mathbf{e}_{2}$, which in turn depend on the nature of the original states. Let us consider a specific example. Assume that the radiators are atoms having a ${ }^{1} P_{1}$ excited state and a ${ }^{1} S_{0}$ ground state. Assume that the degeneracy of the excited state is split by a magnetic field in the $z$ direction and that the $m_{l}=1$ excited level is being used. Under these conditions $\mathbf{e}_{1}$ and $\mathbf{e}_{2}$ are orthogonal to each other and the $z$ axis, and the system has energy levels and interactions with the field identical with those of a spinning top having an electric dipole moment along its axis and precessing about the $z$ axis as a result of an interaction with a static electric field in that direction. Consequently, since large quantum numbers are involved, to a good approximation the gas can be replaced by this classical model, which consists of a spinning top, in calculating both the interaction of the field on the gas and vice versa.

\footnotetext{
${ }^{11}$ Reference 1, p. 26.
}

\section{RADIATION LINE BREADTH AND SHAPE}

Under conditions for which the above "classical model" is valid, it is easy to calculate the natural line breadth and shape factor. This is of considerable importance in microwave spectroscopy. It has been customary to regard the natural line breadth as too small to be of any practical importance. However, as will be seen below, when coherence is properly taken into account the natural radiation breadth of the line may be far from negligible.

Using the above classical model, the angle between the spin axis and the $z$ axis (the polar angle) will be designated as $\varphi$. In this approximation the quantum number $m$ may be replaced by

$$
m=r \cos \varphi
$$

from which, using Eq. (24), the radiation rate becomes

$$
I=I_{0} r^{2} \sin ^{2} \varphi .
$$

Also, the internal energy of the gas is

$$
m E=r E \cos \varphi \text {. }
$$

Balancing the radiation rate to the energy loss of the gas gives

$$
\begin{aligned}
& \qquad \begin{aligned}
\dot{\varphi}=\left(I_{0} r / E\right) \sin \varphi, & \\
\text { from which, assuming } \varphi & =90^{\circ} \text { if } t=0, \\
\sin \varphi & =\operatorname{sech}(\alpha t),
\end{aligned}
\end{aligned}
$$

where $\alpha=I_{0} r / E$. The radiated wave has the following form as a function of time:

$$
A(t)=\left[\begin{array}{ll}
e^{i \omega t} \sin \varphi, & t>0, \\
0, & t<0,
\end{array} \quad \hbar \omega=E .\right.
$$

The Fourier transform gives the line shape and has the value

$$
Q(\beta)=\left(\frac{\pi}{2}\right)^{\frac{1}{2}} \frac{1}{\alpha} \operatorname{sech}\left(\frac{\pi \beta-\omega}{2} \frac{\beta-}{\alpha}\right) .
$$

It should be noted that this is not of the usual Lorentz form. The line width at half-intensity points is

$$
\Delta \omega=1.12 I_{0} r / E=1.12 \gamma r .
$$

Here $\gamma$ is the line width at half-intensity points for the radiation from isolated single molecules. Putting in the maximum value of $r$ gives a line breadth of $\Delta \omega$ $=1.12 \gamma n / 2$, which is generally very substantially larger than $\gamma$.

\section{RADIATION FROM A GAS OF LARGE EXTENT}

A classical system of simple harmonic oscillators distributed over a large region of space can be so phased relative to each other that coherent radiation is obtained in a particular direction. It might be expected also that the radiating gas under consideration would have energy levels such that spontaneous radiation occurs coherently in one direction. 
It will be assumed that the gas occupies a region having dimensions generally larger than radiation wavelength but small compared with the reciprocal of the natural line width,

$$
\Delta k=\Delta \omega / c
$$

It is necessary to turn again to the general expression for the interaction term in the Hamiltonian equation (13). The vector potential operator can be expanded in plane waves:

$$
\mathbf{A}(\mathbf{r})=\sum_{\mathbf{k}^{\prime}}\left[\mathbf{v}_{\mathbf{k}^{\prime}} \exp \left(i \mathbf{k}^{\prime} \cdot \mathbf{r}\right)+\mathbf{v}_{\mathbf{k}^{\prime}} * \exp \left(-i \mathbf{k}^{\prime} \cdot \mathbf{r}\right)\right]
$$

$\mathbf{v}_{\mathbf{k}^{\prime}}$ and its Hermitian adjoint $\mathbf{v}_{\mathbf{k}^{\prime}}$, are photon destruction and creation operators, respectively. After substituting Eq. (45) into (13), the interaction term becomes

$$
\begin{aligned}
H_{1}=- & \frac{1}{2} \sum_{\mathbf{k}^{\prime}} \mathbf{v}_{\mathbf{k}^{\prime}} \cdot\left(\mathbf{e}_{1}-i \mathbf{e}_{2}\right) \sum_{j=1}^{n} R_{j+} \exp \left(i \mathbf{k}^{\prime} \cdot \mathbf{r}_{j}\right) \\
& -\frac{1}{2} \sum_{\mathbf{k}^{\prime}} \mathbf{v}_{\mathbf{k}^{\prime}} * \cdot\left(\mathbf{e}_{1}+i \mathbf{e}_{2}\right) \sum_{j=1}^{n} R_{j-} \exp \left(-i \mathbf{k}^{\prime} \cdot \mathbf{r}_{j}\right),
\end{aligned}
$$

where $R_{j \pm}=R_{j 1} \pm i R_{j 2}$. In this expression, terms involving the product of the photon creation operator and the "excitation operator" $R_{j+}$, etc., have been dropped as these terms do not lead to first-order transitions for which energy is conserved. The form of Eq. (46) suggests defining the operators:

$$
\begin{aligned}
& R_{\mathrm{k} 1}=\sum_{j}\left(R_{j 1} \cos \mathbf{k} \cdot \mathbf{r}_{j}-R_{j 2} \sin \mathbf{k} \cdot \mathbf{r}_{j}\right), \\
& R_{\mathrm{k} 2}=\sum_{j}\left(R_{j 1} \sin \mathbf{k} \cdot \mathbf{r}_{j}+R_{j 2} \cos \mathbf{k} \cdot \mathbf{r}_{j}\right) .
\end{aligned}
$$

In terms of these operators the interaction energy becomes

where

$$
H_{1}=-\frac{1}{2} \sum_{\mathrm{k}^{\prime}}\left(\mathbf{v}_{\mathrm{k}^{\prime}} \cdot \mathbf{e} R_{\mathrm{k}^{\prime}+}+\mathbf{v}_{k^{\prime}} \cdot * \mathbf{e}^{*} R_{\mathrm{k}-^{\prime}}\right)
$$

$$
\begin{gathered}
R_{\mathrm{k}^{\prime} \pm}=R_{\mathrm{k}^{\prime} 1} \pm i R_{\mathrm{k}^{\prime} 2} \\
=\sum_{j=1}^{n} R_{j \pm} \exp \left( \pm i \mathbf{k}^{\prime} \cdot \mathbf{r}_{j}\right) \\
\mathbf{e}=\mathbf{e}_{1}-i \mathbf{e}_{2} .
\end{gathered}
$$

For every direction of propagation $\mathbf{k}$ there are two orthogonal polarizations $\mathbf{v}_{\mathbf{k}}$ of $\mathbf{A}$. By a proper choice of polarization basis, the dot product of one of the basic polarizations with $\mathbf{e}$ can be assumed zero. This radiation oscillator is never excited and can be ignored. The orthogonal polarization is the one which couples with the gas. The polarization of emitted or absorbed radiation is uniquely given by the direction of propagation and need not be explicitly indicated.

The operators of Eq. (47), together with $R_{3}$, obey the angular momentum commutation relations. The operator

$$
R_{\mathrm{k}}{ }^{2}=R_{\mathrm{k1}}{ }^{2}+R_{\mathrm{k} 2}{ }^{2}+R_{3}^{2}
$$

commutes with the operators of Eq. (47) and with $R_{3}$. In Eq. (49) $\mathbf{k}$ is regarded as a fixed index. This operator does not commute with another one of the same type having a different index. Omitting for a moment the translational part of the wave function, wave functions may be so chosen as to be simultaneous eigenfunctions of the internal energy $E R_{3}$ and $R_{\mathrm{k}}{ }^{2}$. They may be written as $\psi_{m r}$ and are generated by an expression analogous to Eq. (21):

$$
R_{\mathrm{k}}^{2} \psi_{m r}=r(r+1) \psi_{m r}, \quad E R_{3} \psi_{m r}=m E \psi_{m r} .
$$

By analogy with the development leading to Eq. (24) it is clear that these states represent correlated states of the gas for which radiation emitted in the $\mathbf{k}$ direction is coherent. Thus, coherence is limited to a particular direction only, provided the initial state of the gas is given by a function of the same type as Eq. (50). The selection rules for the absorption or emission of a photon with momentum $\mathbf{k}$ are

$$
\Delta r=0, \quad \Delta m= \pm 1 .
$$

The spontaneous radiation rate in the direction $\mathbf{k}$ is given by Eq. (24), where $I$ and $I_{0}$ are now to be interpreted as radiation rates per unit solid angle in the direction $\mathbf{k}$. This may be written as

$$
I(\mathbf{k})=I_{0}(\mathbf{k})[(r+m)(r-m+1)] .
$$

If a photon is emitted or absorbed having a momentum $\mathbf{k}^{\prime} \neq \mathbf{k}$, the selection rules are

$$
\Delta r= \pm 1,0 ; \quad \Delta m= \pm 1 .
$$

To prove this, it may be noted that the commutation relations of the $2 n$ operators

$$
\begin{aligned}
& R_{j 1}{ }^{\prime}=R_{j 1} \cos \left(\mathbf{k} \cdot \mathbf{r}_{j}\right)-R_{j 2} \sin \left(\mathbf{k} \cdot \mathbf{r}_{j}\right), \\
& R_{j 2}{ }^{\prime}=R_{j 1} \sin \left(\mathbf{k} \cdot \mathbf{r}_{j}\right)+R_{j 2} \cos \left(\mathbf{k} \cdot \mathbf{r}_{j}\right),
\end{aligned}
$$

with those of Eq. (47) are of the same type as denoted by Condon and Shortley ${ }^{12}$ as T. The selection rules satisfied by these operators are of the type given by Eq. (52).$^{13}$ The operators of Eq. (47), with $\mathbf{k}=\mathbf{k}^{\prime}$, may be expressed as linear combinations of those of $\mathrm{Eq}$. (53). Hence the operators of Eq. (47), with $\mathbf{k}$ replaced by $\mathbf{k}^{\prime}$, satisfy the selection rules given by Eq. (52).

As was discussed previously in the dipole approximation, super-radiant states may be excited by irradiating the gas with radiation until states in the vicinity of $m=0$ are excited. In the present case the incident radiation is assumed to be plane with a propagation vector $\mathbf{k}$. After excitation the gas radiates coherently in the $\mathbf{k}$ direction. Because of the selection rules Eq. (52), radiation in directions other than $\mathbf{k}$ tends to destroy the coherence with respect to the direction $\mathbf{k}$ by causing transitions generally to states of lower $r$.

\section{DOPPLER EFFECT}

Because of the occurrence of the center-of-mass coordinates in the "cooperation" operator Eq. (49), it fails to commute with $H_{0}$ [Eq. (1)]; hence eigenstates of $R_{\mathrm{k}}{ }^{2}$ are generally not stationary. This is equivalent to the fact that relative motion of classical oscillators will gradually destroy the coherence of the emitted radiation. If, on the other hand, a set of classical oscillators all move with the same velocity, the state of coherence

\footnotetext{
${ }^{12}$ Reference 6, p. 59.

${ }^{13}$ Reference 6, pp. 60-61.
} 
is stationary. The corresponding question in the case of the quantum mechanical system is whether there exist simultaneous eigenstates of $H$ and $R_{\mathrm{k}}{ }^{2}$ such that coherent radiation is emitted in a transition from one state to another. By starting with the state defined by

$$
\psi_{\mathrm{s} r r}=\left(\exp i \mathbf{s} \cdot \sum_{j} \mathbf{r}_{j}\right) \cdot[+++\cdots+], \quad r=n / 2,(54)
$$

and using the method leading to Eq. (21), there is obtained the set of states

$$
\psi_{\mathrm{s} m r}=\left[\left(R_{\mathrm{k}}{ }^{2}-R_{3}{ }^{2}-R_{3}\right)^{-\frac{1}{2}}\left(R_{\mathrm{k} 1}-i R_{\mathrm{k} 2}\right)\right]^{r-m} \psi_{\mathrm{s} r r} .
$$

If it is assumed that the gas is free, the functions Eq. (55) are simultaneous eigenfunctions of $H$ and $R_{\mathrm{k}}{ }^{2}$. Consequently, the coherence in the $\mathbf{k}$ direction is stationary.

These states are analogous to the classical oscillators all moving with the same speed. Note one important difference, however; from Eq. (55) the momentum of an excited molecule is always

$$
\mathbf{p}_{+}=\hbar \mathbf{s} \text {, }
$$

whereas if a molecule is in its ground state the momentum, as given by Eq. (55), is

$$
\mathbf{p}_{-}=\hbar(\mathbf{s}-\mathbf{k}) \text {, }
$$

the difference being the recoil momentum of the photon. Thus, the coherent states Eq. (55) are always a superposition of states such that the excited molecules have one momentum and the unexcited have another. Hence it is clear that the recoil momentum given to a molecule when it radiates in the $\mathbf{k}$ direction does not produce a molecular motion which destroys the coherence but rather is required to preserve the coherence.

The gain or loss in photon energy which has its origin in the Doppler effect is equal to the loss or gain in the kinetic energy of a radiator which results from the photon-induced recoil. Expressed as a fractional shift in photon frequency, this is

$$
\frac{\Delta \omega}{\omega}=\frac{\hbar\left(\mathbf{S}-\frac{1}{2} \mathbf{k}\right) \cdot \mathbf{k}}{M c k} .
$$

Here $M$ is the molecular mass. For energy states such that $|m| \ll n / 2$, Eq. (58) can be written as

$$
\frac{\Delta \omega}{\omega}=\frac{\mathbf{v} \cdot \mathbf{k}}{c k} \text {. }
$$

Where $\mathbf{v}$ is the total momentum of the gas divided by its total mass. Equation (59) is the usual classical expression for the Doppler shift for a radiator moving with a velocity $\mathbf{v}$. Consequently, for the highly correlated states $|m| \sim 0$ the Doppler effect can be described in classical terms.

The stationary states Eq. (55) do not form a complete set. In particular, the final state, a photon being emitted or absorbed with a momentum not $\mathbf{k}$, is not one of these states. The set of stationary states may be made complete by adding all the other possible orthogonal plane wave states, each being characterized by a definite momentum and internal energy for each molecule. With this set of orthogonal states, matrix elements can be easily calculated for transitions from the states given by Eq. (55) to states in which photons appear having momenta not equal to $\mathbf{k}$. These matrix elements are found to have a magnitude characteristic of the incoherent radiation process. It should be noted that only for one magnitude of $\mathbf{k}$ as well as for direction are the matrix elements of a coherent transition obtained.

\section{PULSE-INDUCED COHERENCE RADIATION}

It will be assumed in this section that a gas initially in thermal equilibrium is illuminated for a short time by an intense radiation pulse. The intensity and angular dependence of the spontaneous radiation emitted after the pulse will be calculated. In order to avoid the difficulties associated with motional effects, the molecules will be assumed so massive that their center-of-mass coordinates can be represented by small stationary wave packets. The center-of-mass coordinates will be then treated as time-independent parameters in the equation. It is assumed that the intensity of the exciting radiation pulse is so great that the fields acting on the gas during the pulse can be considered as described classically. The spontaneous radiation rate after the exciting pulse will be calculated quantum mechanically.

Because the initial state of the gas is a mixed state describing thermodynamic equilibrium, it is convenient to use the density matrix formalism. ${ }^{14}$ It will be assumed that one has an ensemble of gas systems statistically identical and that what one is calculating is certain ensemble averages.

For a pure state, Eq. (28) shows that the spontaneous radiation rate in the $\mathbf{k}^{\prime}$ direction can be written as the expectation value

$$
I\left(\mathbf{k}^{\prime}\right)=I_{0}\left(\mathbf{k}^{\prime}\right)\left\langle R_{\mathrm{k}^{\prime}+} R_{\mathrm{k}^{\prime}-}\right\rangle .
$$

For a state which may be mixed or pure using the density matrix formalism this becomes the trace

$$
I\left(\mathbf{k}^{\prime}\right)=I_{0}\left(\mathbf{k}^{\prime}\right) \operatorname{tr} R_{\mathrm{k}^{\prime}-\rho} R_{\mathbf{k}^{\prime}+} .
$$

Here the density matrix is defined as the ensemble mean

$$
\rho=\left[\psi \psi^{*}\right]_{\mathrm{Av}} \text {. }
$$

In Eq. (63) the wave function $\psi$ is interpreted as a column vector and the $*$ is the Hermitian adjoint. The symbol [ ] Av signifies an ensemble mean.

Assume that the exciting radiation pulse is in the form of a plane wave in the $\mathbf{k}$ direction. The fields which act on the various molecules differ only in their arrival time. The Hamiltonian of the system can be written

$$
H=\hbar \omega R_{3}-\sum_{j} \mathbf{A}_{j}(t) \cdot\left(\mathbf{e}_{1} R_{j 1}+\mathbf{e}_{2} R_{j 2}\right) .
$$

Here $\mathbf{A}_{j}(t)$ is a classical field quantity and

$$
\begin{array}{ll}
\mathbf{A}_{j}(t)=0, & t<t_{j}, \\
& t>t_{j}+\tau
\end{array}
$$

\footnotetext{
${ }^{14}$ R. C. Tolman, The Principles of Statistical Mechanics
} (Clarendon Press, Oxford, 1938), p. 325. 
where $t_{j}$ is the arrival time of the radiation pulse at the $j$ th molecule. Neglecting for the moment the interaction term, the time dependence of the wave function can be given by the unitary transformation

$$
\psi(t)=\exp \left(-i \omega t R_{3}\right) \cdot \psi(0) .
$$

In general, the wave function after the interaction with the electromagnetic field can be obtained through a unitary transformation on the wave function prior to the pulse. The wave function of the gas after the radiation pulse has passed completely over the gas can be related to that before by

$$
\psi^{\prime}(t)=\exp \left(-i \omega t R_{3}\right) T \psi(0)
$$

Here $T$ is a unitary matrix which represents the effect of the pulse on the gas. To find the most general form of $T$ it is convenient to consider the effect of the pulse on a particular molecule. Since this molecule has only two internal states of interest, its wave function can be regarded as a spinor in a pseudo "spin space." Then, apart from a multiplicative phase factor which has no physical significance, any unitary transformation can be represented as a rotation in "spin space." Any arbitrary rotation can be represented as a rotation about the No. 3 axis followed by a rotation about an axis perpendicular to No. 3. Except for the arrival time the radiation pulse is identical in its effect on each molecule of the gas. The operator $T$ can be written then as the product

$$
\begin{aligned}
& T=\exp \left[i \omega \sum_{j} t_{j} R_{j 3}\right] \\
& \cdot \prod_{l} \exp i\left[\begin{array}{l}
\theta \\
\left.\frac{\theta}{2}\left(R_{l+} \alpha+R_{l \_} \alpha^{*}\right)+\theta^{\prime} R_{l 3}\right] \\
\cdot \exp \left[-i \omega \sum_{j} t_{j} R_{j 3}\right] .
\end{array}\right.
\end{aligned}
$$

The first and second rotations are through angles of $\theta^{\prime}$ and $\theta$, respectively, and the phase of $\alpha$ determines the direction of the 2 nd rotation axis. It is assumed that $|\alpha|=1$ and that the arrival time at the $j$ th molecule is

$$
t_{j}=(1 / \omega) \mathbf{k} \cdot \mathbf{r}_{j}
$$

Equation (67a) becomes Eq. (68) after making use of (67b):

$$
T=\underset{2}{\exp i-}\left(R_{\mathrm{k}+\alpha}+R_{\mathrm{k}-\alpha^{*}}\right) \cdot \exp i \theta^{\prime} R_{3} .
$$

It should be noted that the effect of the different times of arrival of the pulse at the various molecules is contained in $\mathbf{k} \cdot \mathbf{r}_{j}$ which appears in $R_{\mathrm{k}_{ \pm}}$in Eq. (68).

The reason for choosing this transformation to be a rotation about No. 3 followed by a perpendicular rotation is that the rotation about No. 3 is the same as a time displacement and has no effect since the initial state is assumed to be one of thermal equilibrium.

Assume that the initial density matrix can be written as

$$
\begin{gathered}
\rho_{0}=\frac{\exp \left(-E R_{3} / k T\right)}{\operatorname{tr} \exp \left(-E R_{3} / k T\right)}=2^{-n} \prod_{j}\left(1-\gamma R_{j 3}\right), \\
\gamma=2 \tanh (E / 2 k T)
\end{gathered}
$$

The density matrix after the radiation pulse is

$$
\rho(t)=\exp \left(-i \omega t R_{3}\right) \cdot T \rho_{0} T^{-1} \exp \left(i \omega t R_{3}\right) .
$$

The spontaneous radiation rate after the exciting pulse is given by Eq. (62) which becomes

$$
I\left(\mathbf{k}^{\prime}\right)=I_{0}\left(\mathbf{k}^{\prime}\right) \operatorname{tr} T \rho_{0} T^{-1} R_{\mathrm{k}^{\prime}+} R_{\mathbf{k}^{\prime}-},
$$

since $R_{3}$ commutes with $R_{\mathrm{k}^{\prime}+} R_{\mathrm{k}^{\prime}-}$. The radiation rate is thus independent of the time after the exciting pulse. This is because the effect of the radiated field on the gas has been neglected. Equation (71) is to be interpreted as the radiation rate immediately after the exciting pulse. Since $\rho_{0}$ and $R_{3}$ commute, Eq. (71) can be written as

$$
\begin{aligned}
& I\left(\mathbf{k}^{\prime}\right)=I_{0}\left(\mathbf{k}^{\prime}\right) \operatorname{tr} \exp \left[\frac { 1 } { 2 } i \theta \left(R_{\mathrm{k}+} \alpha+R_{\left.\left.\mathrm{k}-\alpha^{*}\right)\right] \cdot \rho_{0}}\right.\right. \\
& \cdot \exp \left[-\frac{1}{2} i \theta\left(R_{\mathrm{k}+} \alpha+R_{\mathrm{k}-} \alpha^{*}\right)\right] \cdot R_{\mathrm{k}^{\prime}+} R_{\mathrm{k}^{\prime}} .
\end{aligned}
$$

It is desirable to transform $\rho_{0}$ before evaluating the trace

$$
\begin{aligned}
& \rho^{\prime}=\exp \left[\frac{1}{2} i \theta\left(R_{\mathrm{k}+} \alpha+R_{\mathrm{k}-} \alpha^{*}\right)\right] \\
& \cdot \rho_{0} \exp \left[-\frac{1}{2} i \theta\left(R_{\mathrm{k}+} \alpha+R_{\mathrm{k}-} \alpha^{*}\right)\right] \\
& \quad=2^{-n} \prod_{j}\left(1-\gamma R_{j 3}^{\dagger}\right) \\
& \text { where } \quad R_{j 3}^{\dagger}=R_{j 3} \cos \theta-\frac{1}{2} i\left(R_{j+}^{\prime} \alpha-R_{j-}^{\prime} \alpha^{*}\right) \sin \theta
\end{aligned}
$$

The primed operators are obtained from Eq. (53) as

$$
R_{j \pm}{ }^{\prime}=R_{j 1}{ }^{\prime} \pm i R_{j 2}{ }^{\prime}=R_{j \pm} \exp \left( \pm i \mathbf{k} \cdot \mathbf{r}_{j}\right)
$$

The trace in Eq. (72) can now be evaluated to give

$$
I\left(\mathbf{k}^{\prime}\right)=I_{0}\left(\mathbf{k}^{\prime}\right) \sum_{j l} \operatorname{tr} 2^{-n} \prod_{s}\left(1-\gamma R_{s 3^{\dagger}}{ }^{\dagger}\right) R_{j+}{ }^{\prime \prime} R_{l-}{ }^{\prime \prime} .
$$

The double prime is Eq. (75) referred to the $\mathbf{k}^{\prime}$ direction. To evaluate the trace the following relations are needed: For $A_{i}$ and $B_{j}$ functions of the $R$ 's of molecules $i$ and $j$,

$$
\begin{gathered}
\operatorname{tr} A_{i} B_{j}=2^{--n} \operatorname{tr} A_{i} \operatorname{tr} B_{j}, \\
\operatorname{tr} R_{j 3}=\operatorname{tr} R_{j \pm}=0, \quad \operatorname{tr} R_{j 3}{ }^{2}=2^{n-2}, \\
\operatorname{tr} R_{j+} R_{j-}=\operatorname{tr} R_{j} \_R_{j+}=2^{n-1} .
\end{gathered}
$$

The final result is

$$
\begin{aligned}
& I\left(\mathbf{k}^{\prime}\right)=I_{0}\left(\mathbf{k}^{\prime}\right) \cdot \frac{1}{2} n[1-\cos \theta \cdot \tanh (E / 2 k T) \\
&+\frac{1}{2} \sin ^{2} \theta \cdot \tanh ^{2}(E / 2 k T) \\
&\left.\cdot\left(n\left|\left[\exp i\left(\mathbf{k}-\mathbf{k}^{\prime}\right) \cdot \mathbf{r}\right]_{\mathrm{AV}}\right|^{2}-1\right)\right] .
\end{aligned}
$$

Here the symbol []$_{A v}$ signifies a mean over all the molecules of the gas. For the example considered in Eq. (37a) this mean is unity, and Eq. (37a) follows by integrating over all directions of the emitted radiation. Aside from the factor $I_{0}\left(\mathbf{k}^{\prime}\right)$, the directional dependence of the emitted radiation is given by this mean. This factor is identical with the distribution factor for radiation about a set of classical isotropic radiators which have been excited by a plane wave. Consequently, for a $\theta$ of $90^{\circ}$ and $n \tanh ^{2}(E / k T)$ large compared with unity, the angular distribution of radiation is just the classical one.

The physical significance of the angle $\theta$ is that $\sin 2 \frac{1}{2} \theta$ 
is the probability of the pulse exciting a molecule in its ground state. Also, if the exciting pulse is a constant amplitude wave of frequency $\omega$ during the duration of the pulse, the angle $\theta$ is proportional to the product of pulse amplitude and duration.

If the radiating system consists of a set of particles of spin $\frac{1}{2}$ in a uniform magnetic field, the angle $\theta$ has a geometrical significance. The initial state of a particle will have spin parallel or antiparallel to the field. The radiofrequency pulse will change its state such that its spin axis will be tipped through an angle $\theta$. Note that if $\theta=180^{\circ}$ the populations of the + and - populations have been just interchanged, corresponding to a transition from a positive temperature $T$ to the negative temperature $-T \cdot{ }^{15} \theta=90^{\circ}$ corresponds to the excitation of molecules to energy superposition states Eqs. (38) and (39) for which the gas is radiating coherently.

\section{ANGULAR CORRELATION OF SUCCESSIVE PHOTONS}

The system to be considered here is assumed to be initially in thermal equilibrium. It is allowed to radiate spontaneously. The angular correlation between successive photons is calculated. This correlation was implicit in some of the earlier development, for example in Eq. (51a). As an example, consider a gas composed of widely separated molecules, all excited. Assume that a photon is emitted in the $\mathbf{k}$ direction. The radiation rate for the second photon in this direction is by Eq. (51a).

$$
I(\mathbf{k})=I_{0}(\mathbf{k}) 2(n-1) .
$$

This is twice the incoherent rate. It is not hard to show that for an intermolecular spacing large compared with a radiation wavelength the radiation rate averaged over all directions is the incoherent rate. Hence from Eq. (79) the radiation probability in the direction $\mathbf{k}$ has twice the probability averaged over all directions.

In the problem to be considered, the system will consist initially of the gas in thermal equilibrium having a temperature $T$ (possibly negative) and a photonless field. The molecules will be assumed fixed in position and with intermolecular distances large compared with a radiation wavelength. Photons are observed to be emitted in the directions $\mathbf{k}_{1}, \mathbf{k}_{2}, \cdots, \mathbf{k}_{s-1}$ and only these photons are emitted. The problem is one of finding the radiation rate in the $\mathbf{k}_{s}$ direction for the next photon.

Stated more exactly, it is assumed that there is an ensemble of gaseous systems, each with its own external radiation field. Every member of the ensemble which is capable of radiating will eventually radiate a photon. Those members which radiate their first photon into a small solid angle in the direction $\mathbf{k}_{1}$, are selected to form a new ensemble. For this second ensemble the time zero is taken to be the time that a photon was detected for each member of the ensemble.

It is convenient to calculate correlations for the gas systems forming a microcanonical distribution having an energy per gas system of $m_{0} E$. The results for a

\footnotetext{
${ }^{15}$ E. M. Purcell and R. V. Pound, Phys. Rev. 81, 279 (1951).
}

canonical distribution with a temperature $T$ can subsequently be determined as an average over the microcanonical distributions.

Since the initial state of the system is assumed photonless, it is sufficient to give the explicit dependence of the initial density matrix on the molecular coordinates. Except for normalization this can be written as a projection operator for states of molecular energy $m_{0} E$. A particularly useful form for this density matrix is

$$
\rho_{0}=\frac{\sum_{q=1}^{n} \exp 2 \pi i \frac{q}{n}\left(R_{3}-m_{0}\right)}{\operatorname{tr} \sum_{q=1}^{n} \exp 2 \pi i \frac{q}{n}\left(R_{3}-m_{0}\right)} .
$$

This is a convenient way to write the density matrix because of the relation

$$
\begin{aligned}
\exp \left(\underset{n}{2 \pi i-R_{3}}\right) & =\prod_{i} \exp \left(\underset{n}{2 \pi i-R_{j 3}}\right) \\
& =\prod_{i}\left[\cos \left(\frac{q}{n}-\right)+2 i R_{j 3} \sin \left(\frac{q}{n}\right)\right] .
\end{aligned}
$$

Here the product is over $j=1, \cdots, n$. To illustrate the importance of Eq. (81) the trace appearing in the denominator $D$ of Eq. (80), will be calculated using the relations Eq. (77).

$$
\begin{aligned}
& D=\sum_{q=1}^{n} \exp \left(-2 \pi \underset{n}{q}-m_{0}\right) \cdot \operatorname{tr} \prod\left[\cos \left(\begin{array}{c}
q \frac{q}{n} \\
n
\end{array}\right)\right. \\
& \left.+2 i R_{j 3} \sin \left(\frac{q}{n}\right)\right] \\
& =\sum_{q=1}^{n} 2^{n} \exp \left(\underset{n}{-2 \pi i-m_{0}}\right) \cdot \cos ^{n}\left(\begin{array}{c}
\pi \frac{q}{n} \\
n
\end{array}\right) \\
& =\frac{n ! n}{\left(\frac{1}{2} n+m_{0}\right) !\left(\frac{1}{2} n-m_{0}\right) !}, \quad\left|m_{0}\right|<\frac{n}{2} \\
& =2 n \text { for }\left|m_{0}\right|=n / 2 \text {. }
\end{aligned}
$$

After one photon has been emitted and absorbed in the photon detector, the system is again photonless and its density matrix is (see Appendix 1)

$$
\rho_{1}=\left(R_{\mathrm{k}_{1}-\rho_{0}} R_{\mathrm{k}_{1}+}\right) /\left(\operatorname{tr} R_{\mathrm{k}_{1}-\rho_{0}} R_{\mathrm{k}_{1}+}\right) .
$$

After $s-1$ photons it is

$$
\rho_{s-1}=\frac{R_{\mathrm{k}_{s-1}}-\cdots R_{\mathrm{k}_{1}-\rho_{0}} R_{\mathrm{k}_{1}+} \cdots R_{\mathrm{k}_{s-1}+}}{\operatorname{tr} R_{\mathrm{k}_{s-1}-} \cdots R_{\mathrm{k}_{1}-\rho_{0}} R_{\mathrm{k}_{1}+} \cdots R_{\mathrm{k}_{s-1}+}} .
$$

The $R$ 's are defined in Eqs. (48) and (47) or (46). The radiation rate in the $\mathbf{k}_{s}$ direction immediately after the $s-1$ photon is from Eq. (62)

$$
I\left(\mathbf{k}_{s}\right)=I_{0}\left(\mathbf{k}_{s}\right) \operatorname{tr} R_{\mathbf{k}_{s}-\rho_{s-1}} R_{\mathbf{k}_{s}}+.
$$

Note that $s \leqslant \frac{1}{2} n+m_{0}$. For any $l, R_{l \pm}{ }^{2}=0$. Consequently, 
the numerator of Eq. (84) can be written

$$
\begin{gathered}
\frac{1}{(s-1) !} \sum_{u, v \cdots=1}^{s-1} \sum_{u^{\prime}, v^{\prime} \cdots=1}^{s-1} \sum_{j, l \cdots=1}^{n} \\
\times \exp i\left[\left(\mathbf{k}_{u}-\mathbf{k}_{u^{\prime}}\right) \cdot \mathbf{r}_{j}+\left(\mathbf{k}_{v}-\mathbf{k}_{v^{\prime}}\right) \cdot \mathbf{r}_{l}+\cdots\right] . \\
R_{j-} R_{l} \cdots \rho_{0} \cdots R_{l+} R_{j+} .
\end{gathered}
$$

Each of the above sums is over $s-1$ indices, including only terms for which all $s-1$ indices take on different values. The trace of the expression appears in the denominator of Eq. (84). In order to evaluate this trace it is necessary first to evaluate

$$
\begin{array}{r}
\operatorname{tr} R_{j-} R_{l-} \cdots \rho_{0} \cdots R_{l+} R_{j+}=\operatorname{tr} \rho_{0} \cdots R_{l+} R_{l} R_{j+} R_{j-} \\
=\operatorname{tr} \rho_{0} \cdots\left(\frac{1}{2}+R_{l 3}\right)\left(\frac{1}{2}+R_{j 3}\right) .
\end{array}
$$

If Eqs. (80), (81), and (82) are substituted into Eq. (87), and use is made of Eq. (77) and the equality

$\operatorname{tr}\left[\cos (\pi q / n)+2 i R_{j 3} \sin (\pi q / n)\right]\left(\frac{1}{2}+R_{j 3}\right)$

Eq. (87) becomes

$$
=2^{n-1} \exp (i \pi q / n) \text {, }
$$

$$
\begin{aligned}
& =\frac{2^{n-s+1}}{D} \sum_{q=1}^{n} \exp \left[i \pi \frac{q}{n}\left(s-1-2 m_{0}\right)\right] \cdot \cos ^{n-s+1}\left(\begin{array}{c}
q-\frac{q}{n} \\
n
\end{array}\right) \\
& =\frac{(n-s+1) !\left(\frac{1}{2} n+m_{0}\right) !}{n !\left(\frac{1}{2} n+m_{0}-s+1\right) !}, \quad\left|m_{0}\right|<\frac{1}{2} n \text { or }\left|m_{0}\right|=\frac{1}{2} n, s=1 \\
& =\frac{1}{2}, \quad\left|m_{0}\right|=\frac{1}{2} n, s>1 .
\end{aligned}
$$

Making use of Eq. (88) the denominator of Eq. (84) can be written as

$$
\begin{array}{r}
=P_{s-1} \frac{(n-s+1) !\left(\frac{1}{2} n+m_{0}\right) !}{n !\left(\frac{1}{2} n+m_{0}-s+1\right) !}, \begin{array}{r}
\left|m_{0}\right|<\frac{1}{2} n \quad \text { or } \\
\left|m_{0}\right|=\frac{1}{2} n, \quad s=1
\end{array} \\
=\frac{1}{2} P_{s-1}, \quad m_{0}=\frac{1}{2} n, s>1,
\end{array}
$$

where

$$
\begin{gathered}
P_{s-1}=\frac{1}{(s-1) !} \sum_{u, v \cdots=1}^{s-1} \sum_{u^{\prime}, v^{\prime} \cdots=1}^{s-1} \sum_{j, l}^{n} \\
\times \exp i\left[\left(\mathbf{k}_{u}-\mathbf{k}_{u^{\prime}}\right) \cdot \mathbf{r}_{j}+\left(\mathbf{k}_{v}-\mathbf{k}_{v^{\prime}}\right) \cdot \mathbf{r}_{l}+\cdots\right], \quad s>1 \\
P_{0}=1 .
\end{gathered}
$$

Here, as before, each of the above sums is over $s-1$ indices, including only terms for which all $s-1$ indices take on different values. If Eq. (84) is substituted into Eq. (85), the numerator is Eq. (89) with $s$ increased by one unit. Consequently, substituting Eq. (89) into Eq. (85),

$$
I\left(\mathbf{k}_{s}\right)=I_{0}\left(\mathbf{k}_{s}\right) \frac{P_{s}\left(\frac{1}{2} n+m_{0}-s+1\right)}{P_{s-1}(n-s+1)} .
$$

To restate the meaning of this equation, $I\left(\mathbf{k}_{s}\right)$ is the radiation probability per unit time per unit solid angle in the direction $\mathbf{k}_{s} ; I_{0}\left(\mathbf{k}_{s}\right)$ is the corresponding radiation probability for a single isolated excited molecule. It has been assumed that the gas was initially in the energy state $m_{0} E$ [see Eq. (3)] with a random distribution over the degeneracy of this state. The gas was observed to radiate photons $\mathbf{k}_{1}, \mathbf{k}_{2}, \cdots \mathbf{k}_{s-1}$ previously to $\mathbf{k}_{s}$. Equation (91) is the radiation rate immediately after the $\mathbf{k}_{s-1}$ photon was observed. As a check on the correctness of this expression, note that the incoherent rate is obtained if $s=1$. Also, for $m_{0}=\frac{1}{2} n$ and $\mathbf{k}_{1}=\mathbf{k}_{2}=\cdots$ $=\mathbf{k}_{s}=\mathbf{k}$, the radiation rate Eq. (91) agrees with Eq. (51a).

It should be noted that Eq. (91) is independent of the ordering of the subscripts $1, \cdots, s-1$. Consequently, the angular distribution of the $s$ photon is dependent upon the direction of a previous photon but is independent of the previous photon's position in the sequence of prior photons.

For a gas which contains a large number of randomly positioned molecules and for which previous photons have either been emitted in the direction $\mathbf{k}_{3}$ or in quite different directions, the radiation rate $[\mathrm{Eq} .(91)]$ is approximately equal to the incoherent rate times the number of photons previously emitted in this direction plus one.

Perhaps the case of most physical interest is where $s=2$. In this case Eq. (91) becomes

$$
\begin{gathered}
I\left(\mathbf{k}_{2}\right)=I_{0}\left(\mathbf{k}_{2}\right) \frac{\frac{\mathbf{1} n+m_{0}-1}{n-1}}{n-1}\left[n\left|[\exp i \mathbf{\Delta} \mathbf{k} \cdot \mathbf{r}]_{\mathrm{Av}}\right|^{2}+n-2\right], \\
\Delta \mathbf{k}=\mathbf{k}_{2}-\mathbf{k}_{1} \text {. }
\end{gathered}
$$

The symbol []$_{\mathrm{Av}}$ signifies an average over all the molecular positions.

In case of a gas system at a temperature $T$, Eq. (91) must be averaged over all possible values of $m_{0}$ to give

$$
\bar{I}\left(\mathbf{k}_{s}\right)=I_{0}\left(\mathbf{k}_{s}\right) \frac{P_{s} \sum_{m_{0}=s-\frac{1}{2} n-1}^{\frac{1}{2} n}\left(\frac{1}{2} n+m_{0}+1-s\right) \frac{n !}{\left(\frac{1}{2} n+m_{0}\right) !\left(\frac{1}{2} n-m_{0}\right) !} \exp \left(-\frac{m_{0} E}{k T}\right)}{(n-s+1) P_{s-1} \sum_{m_{0}=s-\frac{1}{2} n-1}^{\frac{1}{2} n} \frac{n ! \exp \left(-m_{0} E / k T\right)}{\left(\frac{1}{2} n+m_{0}\right) !\left(\frac{1}{2} n-m_{0}\right) !}} .
$$

For $|E / k T| \ll 1$ and $s \ll n$, Eq. (93) can be approxi- where mated by

$$
\bar{I}\left(\mathbf{k}_{s}\right)=I_{0}\left(\mathbf{k}_{s}\right) \frac{\left(\frac{1}{2} n+\bar{m}_{0}+1-s\right) P_{s}}{(n-s+1) P_{s-1}},
$$

$$
\bar{m}_{0}=-\frac{1}{4} n E / k T \text {. }
$$

It is a pleasure to acknowledge the assistance of the author's colleague, Professor A. S. Wightman, who read 
the manuscript and made a number of helpful sug- ment is gestions.

\section{APPENDIX I}

It is assumed that the system consists initially of a gas with an energy $m_{0} E$ and a photonless radiation field. A photon and only one photon is observed to be emitted. The effect of the photon emission on the state of the system is required.

There are two separate effects to be considered. First there is the effect on the state of the system which has its origin in the interaction between the field and gas. Second there is the effect of the observation which determines that a photon and one photon only has been emitted, that this photon was emitted in the $\mathbf{k}$ direction, and that the photon was absorbed in the detector. The first part of the problem is solved using Schrödinger's equation. The Hamiltonian of the system is

$$
\begin{aligned}
H & =\hbar \omega R_{3}+H_{0}+H^{\prime}, \quad H_{0}=\sum_{\mathrm{k}^{\prime}} H_{\mathrm{k}^{\prime}}, \\
H^{\prime} & =-\frac{1}{2} \sum_{\mathrm{k}^{\prime}}\left[\mathbf{v}_{k^{\prime}} \cdot \mathbf{e} R_{\mathrm{k}^{\prime}+}+\mathbf{v}_{\mathrm{k}^{\prime}} * \cdot \mathbf{e}^{*} R_{\mathrm{k}^{\prime}-}\right] .
\end{aligned}
$$

Here $H_{\mathrm{k}^{\prime}}$ is the energy of the $\mathbf{k}^{\prime}$ radiation oscillator. Assume a pure state represented by a wave function $\psi_{0}$ at a time $t=0$. Assume that $\psi_{0}$ is an eigenstate of $R_{3}$ and is photonless. At some later time it is

$\psi(t)=\exp (-i H t / \hbar) \psi_{0}=\left(1-\frac{i}{\hbar} H t-\frac{H^{2}}{2 \hbar^{2}} t^{2}+\cdots\right) \psi_{0}$.

For the quadratic and higher powers of $t$ each term will be a sum of products of $H^{\prime}$ and $\left(H_{0}+\hbar \omega R_{3}\right)$. However, the interaction term $H^{\prime}$ consists of sums of terms of the type

$$
U_{\mathrm{k}^{\prime}}=\mathbf{v}_{\mathrm{k}^{\prime}} \cdot \mathbf{e} R_{\mathrm{k}^{\prime}+}
$$

and its Hermitian adjoint. The operator $U_{\mathbf{k}^{\prime}}$ consists of the product of a photon annihilation operator and a gas excitation operator. It converts an eigenstate of $R_{3}$ and $H_{0}$ into another such or it gives zero. The most general term operating on $\psi_{0}$ in Eq. (96) is therefore a product of powers of $H_{0}+\hbar \omega R_{3}$ and terms of the type $U_{\mathbf{k}^{\prime}}$ and $U_{\mathbf{k}^{\prime}}{ }^{*}$ taken in various orders. In each of these terms $H_{0}+\hbar \omega R_{3}$ always operates on an eigenfunction and consequently can be moved to the end of the product as a number, the eigenvalue. Consequently $\psi(t)$ becomes

$$
\begin{array}{r}
\psi(t)=\left[1+\sum_{\mathbf{k}^{\prime}} g_{\mathbf{k}^{\prime}}(t) U_{\mathbf{k}^{\prime}} *+\sum_{\mathbf{k}^{\prime}} h_{\mathbf{k}^{\prime}}(t) U_{\mathbf{k}^{\prime}} U_{\mathbf{k}^{\prime}} *\right. \\
\left.+\sum_{\mathbf{k}^{\prime} \mathbf{k}^{\prime \prime}} g_{\mathbf{k}^{\prime} \mathbf{k}^{\prime \prime}}(t) U_{\mathbf{k}^{\prime}}{ }^{*} U_{\mathbf{k}^{\prime \prime}} *+\cdots\right] \psi_{0} .
\end{array}
$$

The $g$ 's and $h$ 's are numbers, functions of the time. It may be noted that since $\psi_{0}$ represents a photonless state, an annihilation operator for a given radiation oscillator $\mathbf{k}^{\prime}$ appears only if preceded by the corresponding creation operator.

Assuming that at the time $t$ a photon measurement is made which indicates the presence of photon $\mathbf{k}$ and no other photons, the wave function after the measure-

$$
\psi^{\prime}=P_{\mathrm{k}} \psi,
$$

where the operator $P_{\mathrm{k}}$ is a projection operator for the $\mathbf{k}$ photon state.

$$
P_{\mathrm{k}}=\frac{H_{\mathrm{k}}}{\hbar \omega_{k}} \prod_{\mathrm{k}^{\prime}}\left(\frac{\hbar \omega_{k^{\prime}}-H_{\mathrm{k}^{\prime}}}{\hbar \omega_{k^{\prime}}}\right) .
$$

The product is over all $\mathbf{k}^{\prime} \neq \mathbf{k}$. Two-photon excitation of one radiation oscillator has been neglected.

$$
\begin{aligned}
\psi^{\prime}=\left[g_{\mathrm{k}}(t) U_{\mathrm{k}}{ }^{*}\right. & +\sum_{\mathrm{k}^{\prime}} H_{\mathrm{kk}^{\prime}}(t) U_{\mathrm{k}_{\mathrm{k}}}{ }^{*} U_{\mathrm{k}^{\prime}} U_{\mathrm{k}^{\prime}}{ }^{*} \\
& \left.+\sum_{\mathrm{k}} I_{\mathrm{kk}^{\prime}}(t) U_{\mathrm{k}^{\prime}} U_{\mathrm{k}^{\prime}} * U_{\mathrm{k}^{*}}+\cdots\right] \psi_{0} .
\end{aligned}
$$

In summing over the direction of $\mathbf{k}^{\prime}$ in the second and third terms above, the expression

$$
R_{\mathrm{k}^{\prime}+} R_{\mathrm{k}^{\prime}-}=\sum_{a b} \exp \left[i \mathbf{k}^{\prime} \cdot\left(\mathbf{r}_{a}-\mathbf{r}_{b}\right)\right] \cdot R_{a+} R_{b-}
$$

appears under the integral. By expanding the exponential in spherical harmonics it can be seen that for $a \neq b$ this integral vanishes, as it has been assumed that

$$
\mathbf{k}^{\prime} \cdot\left(\mathbf{r}_{a}-\mathbf{r}_{b}\right) \gg 1 \text { for } a \neq b .
$$

It should be indicated that the angular dependence is not wholly in the exponential in Eq. (102) but exists in part in the square of the dot product of $\mathbf{e}$ and $\mathbf{v}_{\mathbf{k}^{\prime}}$. However, this contribution to the angular dependence includes only spherical harmonics of finite degree in fact with $l<3$. As the only terms which need to be included in Eq. (102) are $a=b$, Eq. (102) becomes

$$
R_{\mathrm{k}^{\prime}+} R_{\mathrm{k}^{\prime}-}=\frac{1}{2}+R_{3}+\text { (terms from } a \neq b \text { ). }
$$

Independent of its position in a series of products of $U$ 's the expression on the right side of Eq. (103) will operate on an eigenfunction and becomes an eigenvalue which can be removed as a number. In the higher-order terms in Eq. (101) $U_{\mathbf{k}^{\prime}}$ and $U_{\mathbf{k}^{\prime}}$, may not appear adjacent to each other, but if they do not, some other pair such as $U_{\mathrm{k}^{\prime \prime}} U_{\mathrm{k}^{\prime \prime}}$ ** will appear, and after removing this as an eigenvalue another such pair will occur, and eventually the $\mathbf{k}^{\prime}$ pair will be adjacent. Consequently, to all orders in the expansion

$$
\psi^{\prime}=f(t) U_{\mathrm{k}}{ }^{*} \psi_{0}
$$

where $f$ is a function of the time of observation. As the photon detector also absorbs the photon, the wave function must be multiplied by the annihilation operator $\mathbf{e} \cdot \mathbf{v}_{\mathbf{k}}$. This gives, except for the time factor,

$$
\psi^{\prime \prime} \sim R_{\mathrm{k}-\psi_{0}},
$$

which is another photonless state but with one quantum less energy.

If the initial density matrix $\rho_{0}$ contains only photonless states of the same energy $m_{0} E$, then from Eqs. (63) and (105) it is transformed to

$$
\rho_{1}=R_{\mathrm{k}-\rho_{0}} R_{\mathrm{k}+} / \operatorname{tr}\left(R_{\mathrm{k}-\rho_{0}} R_{\mathrm{k}+}\right),
$$

representing the photonless state of the ensemble of systems after the emission, detection, and absorption of photon described by $\mathbf{k}$. 